\title{
The Architectural Colour Design Process: An Evaluation of Sequential Media via Semantic Ratings
}

\section{Sibel Ertez Ural, ${ }^{*}$ Semiha Yilmazer}

Department of Interior Architecture and Environmental Design, Faculty of Art, Design and Architecture, Bilkent University, Ankara 06800, Turkey

Received 11 May 2008; revised 21 April 2009; accepted 10 May 2009

\begin{abstract}
In recent studies, contextual situations of applied colours are compared to colours presented as samples or chips. Findings of such studies point out different results in terms of similarities or differences between the evaluations of isolatedlabstract colours and contextualized situations. Architectural and spatial contexts have their own characteristics regarding colouring criteria, so it is of great importance to examine the architectural/spatial colouring process from this point of view. This study explores this process by investigating the consistency of semantic ratings of four sequential stages of the architectural colour design process, namely, colour chips/samples, abstract compositions, perspective drawings and $3 D$ models. The architectural context for the study was a simple interior space. Fifteen different colour schemes were applied on the four media representing the stages. Subjects rated the 15 sets against seven bipolar, five-step semantic differential scales. The scales consisted of harmonious-discord, pleasant-unpleasant, comfortableuncomfortable, spacious-confined, static-dynamic, exciting-calming and extroverted-introverted. Findings indicated that there are significant associations between the evaluations of the abstract compositions, the perspective drawings and the $3 D$ models; however, the evaluations of colour chips are significantly different than the others. The medium effect observed mostly between abstract and contextualized media. Additionally, factor analysis showed that pleasantness, harmony, spaciousness and comfort are connected in the evaluations of contextual situations, while pleasantness and harmony differ from spaciousness and comfort in the evaluations of colour chips and abstract compositions. The factor of activity (arousal)
\end{abstract}

\footnotetext{
*Correspondence to: Sibel Ertez Ural (e-mail: ural@bilkent.edu.tr).
}

(dynamism, excitement, and extroversion) stays the same for all four media. It is also found that different colour characteristics are determinative over different media. (C) 2010 Wiley Periodicals, Inc. Col Res Appl, 35, 343-351, 2010; Published online 22 January 2010 in Wiley InterScience (www. interscience.wiley.com). DOI 10.1002/col.20583

Key words: architectural colour design process; spatial colour; semantic ratings; architectural/spatial context; colour emotions

\section{INTRODUCTION}

In the field of architecture, colour is a design element. It stands as a composition of all its attributes and it exists at a place where all related disciplines overlap. As GreenArmytage says, "successful colour design depends on the designer's knowledge comes from several disciplines and from design itself." 1 Theoretical knowledge comes from several disciplines and arises from research mostly where colours are abstracted and simplified into colour chips and samples. The need for reconsideration of the theoretical knowledge within the architectural context and reallife situations is inevitable.

In design itself, simplifications and abstractions are widely used both in design education and by professionals. Choosing isolated colour samples from various catalogues, developing abstract colour graphics for initial colour decisions and then elaborating on these decisions through various drawings and models seem to be the most common approach to the colour design process. ${ }^{2-4}$ Each of these visualization techniques is progressively used by the participants in the design as a communication medium throughout the process. Hence, corroboration between the different visualization techniques is essential to a reliable colour design method. The presented study deals with different visualization techniques, commonly used during the architectural colour design process, by investigating the consistency of their evaluations via semantic ratings. 


\section{The Colour Design Process in Architecture}

Colour is an element of architecture, thus colour design have to be an integral part of architectural design process. Smith emphasizes that environmental colour is multifaceted, playing a variety of roles in our everyday lives. ${ }^{5}$ However, prototypes and models for designs are often presented in white or monochromatic combinations, irrespective of the materials incorporated and the colours that may be applied in the final constructed building, interior or object. Questions are therefore raised concerning design professionals' perceptions of the importance of colour in relation to space and form, and to the experience of place. According to Mikellides, an architect wants or considers it desirable to use colour in his design on the one hand, but fears or is ignorant of its possibly undesirable effects on people on the other hand. ${ }^{6}$ Mikellides explains this conflict as an architectural dilemma: what we know about colour as scientists and psychologists and what we do as artists and architects. This conflict can only be overcome in architecture by bridging colour theory and application.

This topic continues to be discussed not only in practice but also in education. Janssens and Mikellides investigated architectural students' knowledge about the perceptual and psycho-physiological aspects of colour, colour nomenclature, existing myths and beliefs and how colour is used in their everyday work in the studio. ${ }^{7}$ The findings show there is a severe lack of knowledge about colour research. Reading colour literature and attending lectures or conferences was rare. However, attitudes toward colour research were positive, especially for studies on colour perception and colour preferences. Students perceived colour design as their own future responsibility and basic design education seemed to be the main source of colour information. Although this study is over 10 years old, it is likely that the architectural education has not changed much. According to Akbay, ${ }^{8}$ during the progression of colour education in basic design courses, students' original colour decision tendencies - toward subjective and intuitive attitudesdecreased and their tendencies towards knowledge-based and analytical attitudes increased.

On the other hand, design students are asking for the guidance of a methodology in their colour design, and they need clarification of the ambiguity and generality of the findings of emperical research. At this point, it is important to investigate how to implement theoretical colour knowledge planted via abstract compositions in basic design into the architectural design process. The product of design is coloured. The aim of the designer is to end up with a satisfactory design product supported by a successful colour scheme. Particularly in architecture, aesthetical and functional criteria are determinative over evaluating colour design. The colour design is expected to be compatible with functional appropriateness and requirements, visual comfort and satisfaction of users' needs for sure, nevertheless colour harmony and colour emotions are seemingly the points of departure for achieving a harmonious, pleasing result, spatial quality and desired mood. However, the link between harmony (which is a measure of aesthetics) and pleasantness (which is a measure of emotion) of colour combinations is still being discussed. ${ }^{9}$

Colour Harmony. In architecture, colour is not an isolated entity; it always exists with other colours of the form or the space. Thus, harmony of colour combinations is a major concern for colour designers. On condition that harmony is a "measure of aesthetics", colour harmony is tried to be explained on the order of colours.

According to Arnheim, ${ }^{10}$ the first attempt to define harmonic colour combinations dates back to 1839 , to Chevreul's statement about harmony of similarities and harmony of contrasts. The 20th century has seen several attempts at explain colour harmony. ${ }^{11-14}$ Although these are not guaranteed formulae, they can be considered clues to understanding which colours work well together. These general rules are mostly known as colour schemes, and are broadly based on Chevreul's early statement on colour harmony. According to Burchett, ${ }^{15}$ no acceptable model exists for explaining the concept of colour harmony and the author identifies key attributes and related terms consistently used to describe it.

In recent years, studies of the dynamics of colour combinations and colour harmony have been done using different methods. Hård and Sivik ${ }^{16}$ developed a theoretical model for colour combinations described on the Natural Color System. Nemcsics ${ }^{17}$ examined the harmony content of scales found in different locations in various positions of the axial sections of the Coloroid colour system. Ünver and Öztürk ${ }^{4}$ used contrast quantity limits calculated by the elements of the Munsell Color System. Ou and Lou ${ }^{18}$ developed a quantitative model for two-colour harmony. Ural ${ }^{19}$ examined colour harmony in terms of the colour dynamics of two-colour combinations by analysing the relations between three dimensions of colour. However, there is still no common and generally accepted method for determining the dynamics of colour combinations.

Colour Emotions. Literature on colour emotions has been authored mostly by experimental psychologists. The method of semantic differential introduced by Osgood et $a l .{ }^{20}$ is the generally accepted method for colour emotion research. In this method, results are interpreted from ratings on semantic scales and categories devised by factor analysis. Lack of control of the stimulus material and the viewing conditions, and using isolated colour chips are two points of criticism for such research. From this point of view findings of studies investigating the consistencies and/or contradictions between evaluations of abstract/isolated and contextual situations are of great importance. Such investigations are also carried out by comparing the profiles of semantic ratings and/or the extracted factors obtained from different data sets.

The studies investigating the consistencies and/or contradictions between evaluations of abstract/isolated and contextual situations are compared in detail by $\mathrm{Taft}^{21}$ who states that some of the research supports that colour is an attribute of an object, preferences of colours are 
linked to the objects and colour chip preferences are not generalizable (e.g., Norman and Scott; Wise and Wise; Whitfield and Wiltshire; and Davidoff, cited in $\mathrm{Taft}^{21}$ ) The findings of research which looks at colour-meaning associations in a wider sense rather than merely at preference, point out consistencies between abstract/isolated and contextual situations to some extent. Osgood et al. $^{20}$ found that potency and activity ratings are largely unaffected by the object, while evaluations are dependent on the object. Taft compared semantic ratings of colour samples (chips) with those same colours applied to a variety of familiar objects. ${ }^{18}$ Analyses performed on the data indicated that generally few significant differences existed between chip and object ratings for the same colour. The results of this study have implications for the use of colour chips in colour planning, as colour chips may serve as an economical and expedient medium. In Sivik's ${ }^{22}$ research comparing colour chip ratings with colour ratings of two types of simulated building exteriors, descriptive scales are largely unaffected by context, while evaluative scales are most affected by context. In research by Ural, ${ }^{19}$ a subject group of architects first generated two-colour combinations in an abstract medium and then, after applying those combinations to architecturally defined contexts, subjects were asked to re-evaluate the new situations. Findings showed that subjects generally agreed that the colour combinations had to be reconsidered and they proposed significant colour changes for the new conditions. The findings also showed that contextual characteristics are important components of the decision-making process in architectural colour design. Nevertheless, in an architectural context, space has its own characteristics which are quite different from those that an object has. Billger's article dealt with the way colours in a room affect each other through contrast effects and reflections. ${ }^{23}$ The mentioned study was based on the visual observations of colours in a sequence of experimental situations. It was found that the effects of both contrasts and reflections were evident in the spatial appearance of colours.

In recent research, the use of colour chips has also been examined by making comparisons with the spatial context. $^{24-26}$ Destefani and Whitfield questioned how people select colours for real settings and how they make such aesthetic decisions. ${ }^{24}$ According to the authors, "after more than a century of empirical research in psychology, answers to such elementary question remain elusive and the main reason is that psychology has focused upon questions of theoretical interest within psychology, and used colour as a means to this end. In doing so, typically researchers have investigated people's responses to more abstract stimulimainly colour chips-and neglected colour selection for real-world objects." When Hogg et al. ${ }^{25}$ compared semantic ratings of colour samples and a simulated interior space, they found generally high correlations. However, Stahre et al. $^{26}$ compared the results of a colour-chip study to one of a full-scale room, and found that the colours were perceived as more distinct and stronger and that they aroused much stronger emotions in the full-scale room.

\section{Problems and Aims}

The literature review reveals the fact that theoretical knowledge about colour harmony and colour emotions is based mostly on isolated colour samples and abstract combinations, and it seems that such knowledge needs to be reconsidered within an architectural context. Additionally, during the architectural colouring process, colour chips/samples are widely used at least for initial colour decisions. Research investigating the consistency of colour evaluations in isolated/abstract media and contextual situations in the field of architecture has great importance both for knowledge implementation in the field and for developing architectural colouring methods. Although there is supplementary research that sheds some light on the question, any research investigating the consistency of colour decisions throughout all stages-from isolated chips to abstract media and finally to the form/space simulation(s) - has not been found in the literature.

The aim of this study is thus to analyze the assembly of the stages of the colour design process within an architectural context and to assist and sustain designers/architects by sparking a discussion on colour design method. The scope of the study is circumscribed as comparing the different kinds of visualizations with each other, rather than comparing them with real-life situations. Therefore, this research was designed to explore:

1. Whether the evaluations (semantic ratings) of the colour schemes have associations or differences when the medium changes from colour chips to abstract compositions, from abstractions to contextual situations and from $2 \mathrm{D}$ to $3 \mathrm{D}$ media,

2. Which constructs (semantic scales) go together and how these constructs are categorized (factorized) when the medium that the colour scheme is presented in differs, and

3. Whether the same colour and scheme characteristics predict the evaluations (semantic ratings) of the colour schemes when presented in different media.

\section{METHODS}

To investigate the above research questions, the method of semantic differential was used. In the questionnaire, seven bipolar five-step scaled adjective pairs were applied. For investigating constructs related to an architectural context, evaluative adjectives were preferred and descriptive adjectives were not found to be relevant to the scope and objectives of the research. The chosen adjective pairs were categorized under the three primary factors of Osgood et al. ${ }^{20}$; evaluation, activity and potency (pleasure, arousal and dominance in Mehrabian). ${ }^{27}$ The list of adjectives and the related factors are given in Table I.

Coloured materials used for this research were: colour schemes presented as colour chips, abstract compositions, perspective drawings and 3D models - the sequential tools of colour-design process. These four media were prepared for a simple architectural space $(4 \mathrm{~m} \times 5 \mathrm{~m} \times 3 \mathrm{~m})$ assumed to have a study/work function. 
TABLE I. Bipolar adjectives used in the questionnaire and the related factors identified by Osgood et al. ${ }^{17}$

\begin{tabular}{lll}
\hline & \multicolumn{1}{c}{ Adjectives } & \multicolumn{1}{c}{ Factors } \\
\hline a1 & Harmonious/discord & Evaluation \\
a2 & Pleasant/unpleasant & Evaluation \\
a3 & Comfortable/uncomfortable & Evaluation \\
a4 & Spacious/confined & Potency \\
a5 & Static/dynamic & Potency + activity \\
a6 & Exciting/calming & Activity \\
a7 & Introverted/extroverted & Activity \\
\hline
\end{tabular}

Colour chips were prepared as $3 \mathrm{~cm} \times 3 \mathrm{~cm}$ samples [Fig. 1(a)]. Abstract compositions were generated as a 9 by 9 checkerboard pattern of $3 \mathrm{~cm} \times 3 \mathrm{~cm}$ squares, considering both proportional relations and the adjacency of coloured areas [Fig. 1(b)]. Perspectives were drawn as one point on A4 drawing paper [Fig. 1(c)]. In order not to cause value differences that might affect the appearance of colour schemes, light, shade and shadow effects were eliminated in the perspective drawings. 3D models were produced at a scale of 1/20 [Fig. 1(d)]. For homogeneous illumination and in order to prevent the effects of glare, shade and shadow daylight from the north was the preferred light source.

In an architectural context and particularly in spatial design, the number of colours combined in a scheme differs depending on various factors. In this research, the visual differentiation of surfaces was taken into consideration and the number of colours limited to five. Colours were chosen for the ceiling, floor, back and left walls, right wall and the furniture. In the research, 15 different colour schemes were tested against semantic scales. Scheme characteristics of colour combinations were defined by the three variables of the Munsell Color System (Hue, Value, Chroma). Maximum contrast quantity coefficients ${ }^{4}$ were calculated; coefficients under 0.34 were defined as "similar," coefficients over 0.66 defined as "contrasting" and coefficients between the two defined as "moderate." For the cases defined as "similar," the dominant descriptive characteristics of colours were stated as follows: "warm" or "cold" for Hue, "light" or "dark" for Value, "weak" or "strong" for Chroma. For the cases defined as "contrasting" and "moderate," the descriptive quality was stated as "both" for data analyses. Corresponding Munsell notations, schemes and the dominant descriptive characteristics of the colour combinations can be seen in Table II.

Subjects consisted of 40 second- and third-year interior architecture students, who were asked to evaluate 15 colour schemes presented in four different media. First, subjects were informed about the given space and assumed function, then materials were presented on separate desks and their order changed after each five subjects. In the experiment room, only indirect daylight (from the north) was allowed as illumination. The same viewing and illumination conditions of coloured materials were provided during the experiment. Data consisted of subject ratings on seven bipolar, five-step semantic differential scales of 15 colour schemes, each presented in four different media and colour-scheme characteristics (similarity or contrast and dominant descriptive characteristics over three colour dimensions). Each subject rated a total of 60 cases against all scales. The collected data were analysed by SPSS 8.0 for Windows.

\section{RESULTS}

This research aimed to examine the effects of context throughout the architectural colour-design process by means of different media that, represented the sequential steps of the process. Data analysis based on the three research questions is as follows:

i. To see whether there are any associations between the evaluations of colour schemes presented as chips, abstract colour graphics, perspective drawings and models, data sets were analyzed by correlating the semantic ratings of each medium. Table III shows the correlations (Pearson's $r$ and $P$ ) between colour chips, abstract graphics, perspective drawings and 3D models over seven semantic scales.

Results showed that there is always a significant positive relationship between semantic ratings of three of the media-abstract colour graphics, perspective drawings and models. However, this cannot be said in every case for colour chips. Colour chips showed significant associations with all the other media only for the adjective pairs harmonious/discord and exciting/calming. On the contrary, there is no significant association between chips and

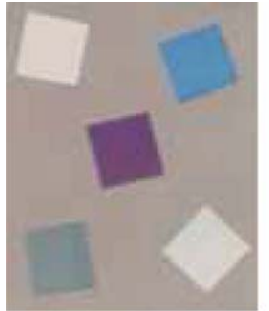

a

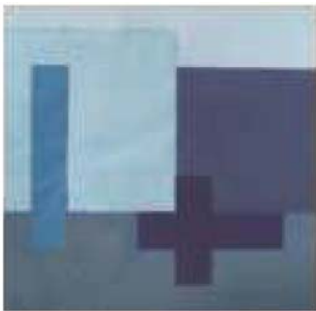

b

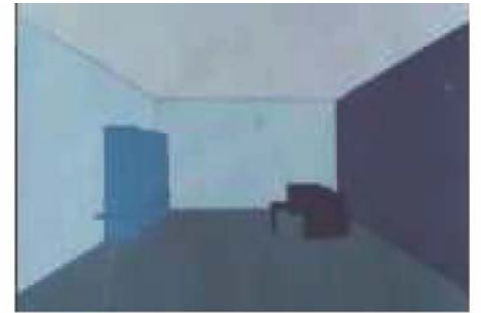

C

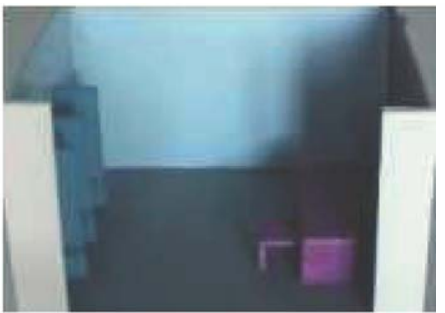

d

FIG. 1. An example of the coloured materials: Colour chips (a), 2D abstract compositions (b), perspective drawings (c), and $3 \mathrm{D}$ models $(\mathrm{d})$. 
TABLE II. Corresponding Munsell notations and scheme/colour characteristics.

\begin{tabular}{|c|c|c|c|c|c|c|c|c|}
\hline Schemes & N1 & N2 & N3 & N4 & N5 & HUE & Value & Chroma \\
\hline 1 & $5 Y 9 / 2$ & $5 Y 9 / 6$ & $2,5 \mathrm{GY} 8 / 3$ & 25GY3/1 & $7,5 Y 8 / 7$ & Similar-warm & Contrasting & Contrasting \\
\hline 2 & $10 Y 9 / 6$ & $7,5 \mathrm{R} 3 / 4$ & 5YR6/14 & $5 R 4 / 12$ & $10 Y 8 / 10$ & Similar-warm & Contrasting & Contrasting \\
\hline 3 & 7,5RP9/2 & 7,5R2/6 & 7,5R6/2 & $6,5 R 4 / 12$ & 7,5RP4/8 & Moderate & Contrasting & Contrasting \\
\hline 4 & $10 \mathrm{~B} 7 / 4$ & $7,5 \mathrm{GY} 8 / 4$ & $5 B 7 / 4$ & $5 P 7 / 6$ & $7,5 B G 7 / 4$ & Contrasting & Similar-light & Similar-weak \\
\hline 5 & $9 / 0$ & $5 \mathrm{~B} 5 / 1$ & $5 \mathrm{~B} 9 / 2$ & $2,5 P 4 / 4$ & $7,5 \mathrm{~B} 5 / 8$ & Similar-cold & Moderate & Contrasting \\
\hline 6 & $5 Y 8,5 / 8$ & $5 Y 8 / 10$ & 3,75YR6/12 & 2,5PB7/8 & $2,5 \mathrm{GY} 7 / 8$ & Contrasting & Similar-light & Similar-strong \\
\hline 7 & $5 Y R 9 / 2$ & $10 R 7 / 8$ & $10 B 8 / 4$ & $10 \mathrm{R} 6 / 12$ & $5 \mathrm{~PB} 5 / 10$ & Contrasting & Similar-light & Contrasting \\
\hline 8 & $5 Y 9 / 2$ & $5 P 8 / 4$ & 5YR9/6 & $5 P 7 / 6$ & $5 Y 8 / 6$ & Contrasting & Similar-light & Similar-weak \\
\hline 9 & $7,5 Y 9 / 2$ & $10 \mathrm{~B} 8 / 4$ & $5 G Y 8 / 6$ & $2,5 \mathrm{~PB} 6 / 10$ & $7,5 Y 9 / 4$ & Contrasting & Similar-light & Contrasting \\
\hline 10 & 10R9/2 & 10R8/4 & 10R6/6 & $7,5 \mathrm{R} 10 / 4$ & 10R5/8 & Similar-warm & Moderate & Moderate \\
\hline 11 & $7,5 B G 8 / 2$ & $7,5 \mathrm{BG} 4 / 2$ & $7,5 B 6 / 6$ & $7,5 \mathrm{BG} 4 / 4$ & 2,5BG $4 / 4$ & Similar-cold & Moderate & Similar-weak \\
\hline 12 & $7 / 0$ & 10YR8/10 & $10 \mathrm{R} 6 / 12$ & $8,75 \mathrm{R} 5 / 12$ & $2,5 Y 8,5 / 12$ & Similar-warm & Similar-light & Contrasting \\
\hline 13 & $5 \mathrm{~B} 7 / 2$ & $5 B 4 / 4$ & $5 P 5 / 6$ & $10 G Y 4 / 6$ & $10 \mathrm{~PB} 4 / 4$ & Contrasting & Similar-dark & Similar-strong \\
\hline 14 & $5 / 0$ & $7,5 \mathrm{P} 5 / 2$ & $7,5 \mathrm{P} 3 / 2$ & $3,75 \mathrm{R} 4 / 12$ & 10RP2/6 & Similar-warm & Similar-dark & Contrasting \\
\hline
\end{tabular}

N1, ceiling; N2, floor; N3, back and left walls; N4, right wall; N5, furniture.

all the other media for the adjective pair pleasing/unpleasing. To investigate medium effect on the semantic ratings of the four media, multiple comparisons in ANOVA (analysis of variance) were performed. Table IV shows the medium effect on the semantic ratings. Results showed an overall significant effect for the type of medium for six of the adjectives. Only for one pair of adjectives (exciting/calming) was no significant effect of medium observed. In Table IV, Scheffe's range shows where the medium effect appears.

Scheffe's range test found that the evaluations of the colour chips differed from the abstract graphics only for the adjective pair introverted/extroverted $(P=0.045)$. Evaluation of the colour chips differed from the perspective drawings for the adjective pairs pleasing/unpleasing $(P=0.013)$, spacious/confined $(P=0.000)$ and comfortable/uncomfortable $(P=0.000)$. Evaluation of the colour chips differed from the model for the adjective pairs comfortable/uncomfortable $(P=0.002)$ and static/dynamic $(P$ $=0.008)$. Semantic ratings of the abstract graphics differed from the perspective drawings for the adjective pairs spacious/confined $(P=0.000)$ and introverted/extroverted $(P=0.000)$. They differed from the model for the adjective pair introverted/extroverted $(P=0.002)$. Differences between the ratings of the perspective drawings and the model appear for the adjective pairs harmonious/discord $(P=0.036)$, spacious/confined $(P=0.028)$, comfortable/ uncomfortable $(P=0.000)$ and static/dynamic $(P=$ 0.042).

ii. Next, factor analysis was performed in order to observe which constructs (semantic scales) go together and how these constructs are categorized (factorized) when the medium that the colour scheme is presented in differs. Table $\mathrm{V}$ shows the variance percentages accounted for the factors and the orthogonal factor loading matrices for seven adjectives of colour chips, abstract graphics, perspective drawings, and 3D models. On the table the four media arranged as columns. Classifications given on top of them show the characteristics of the four media (e.g., colour chips are presenting 2-dimensional, abstract media where colours are loosely combined, while abstract graphics are also 2-dimensional and abstract, but colours are organized as compositions). On the rows the categorization of the constructs are listed under the order of extracted factors.

For the colour chips the first factor accounted for $28 \%$ of the variance, the second factor $26 \%$ and the third factor $25 \%$. The first factor gathers the adjective pairs

TABLE III. Associations among colour chips, abstract graphics, perspective drawings, and $3 \mathrm{~d}$ models over seven semantic scales.

\begin{tabular}{|c|c|c|c|c|c|c|c|c|c|c|c|c|}
\hline & \multicolumn{2}{|c|}{$\begin{array}{c}\text { Chips/abstract } \\
\text { graphics }\end{array}$} & \multicolumn{2}{|c|}{$\begin{array}{l}\text { Chips/ } \\
\text { perspective } \\
\text { drawings }\end{array}$} & \multicolumn{2}{|c|}{ Chips/model } & \multicolumn{2}{|c|}{$\begin{array}{l}\text { Abstract } \\
\text { graphics/ } \\
\text { perspective } \\
\text { drawings }\end{array}$} & \multicolumn{2}{|c|}{$\begin{array}{c}\text { Abstract } \\
\text { graphics/ } \\
\text { model }\end{array}$} & \multicolumn{2}{|c|}{$\begin{array}{l}\text { Perspective } \\
\text { drawings/ } \\
\text { model }\end{array}$} \\
\hline & $r$ & $P$ & $r$ & $P$ & $r$ & $P$ & $r$ & $P$ & $r$ & $P$ & $r$ & $P$ \\
\hline Harmony & 0.279 & $0.000^{\star *}$ & 0.141 & $0.005^{\star}$ & 0.254 & $0.000^{\star *}$ & 0.494 & $0.000^{\star *}$ & 0.464 & $0.000^{* *}$ & 0.440 & $0.000^{\star *}$ \\
\hline Pleasantness & 0.049 & 0.339 & 0.000 & 0.997 & 0.053 & 0.296 & 0.310 & $0.000^{\star *}$ & 0.353 & $0.000^{* *}$ & 0.371 & $0.000^{* *}$ \\
\hline Comfort & 0.161 & $0.001^{\star *}$ & 0.058 & 0.251 & 0.210 & $0.000^{* *}$ & 0.339 & $0.000^{\star \star}$ & 0.332 & $0.000^{\star \star}$ & 0.375 & $0.000^{\star *}$ \\
\hline Spaciousness & 0.239 & $0.000^{\star \star}$ & 0.041 & 0.416 & 0.285 & $0.000^{\star \star}$ & 0.405 & $0.000^{\star \star}$ & 0.456 & $0.000^{\star *}$ & 0.399 & $0.000^{\star *}$ \\
\hline Dynamism & 0.055 & 0.281 & 0.249 & $0.000^{\star \star}$ & 0.221 & $0.000^{* *}$ & 0.323 & $0.000^{\star \star}$ & 0.283 & $0.000^{\star \star}$ & 0.454 & $0.000^{\star *}$ \\
\hline Excitement & 0.243 & $0.000^{\star \star}$ & 0.120 & $0.018^{\star}$ & 0.231 & $0.000^{\star *}$ & 0.361 & $0.000^{\star *}$ & 0.424 & $0.000^{\star *}$ & 0.304 & $0.000^{* *}$ \\
\hline Introversion & 0.116 & $0.022^{\star}$ & 0.084 & 0.096 & 0.159 & $0.002^{\star}$ & 0.365 & $0.000^{\star \star}$ & 0.288 & $0.000^{\star *}$ & 0.358 & $0.000^{\star *}$ \\
\hline
\end{tabular}


TABLE IV. Media effects on semantic ratings of seven adjectives (multiple comparisons in ANOVA).

\begin{tabular}{|c|c|c|c|c|}
\hline & \multirow[b]{2}{*}{$F$} & \multirow[b]{2}{*}{$P$} & \multicolumn{2}{|l|}{ Scheffe's range } \\
\hline & & & Medium & $P$ \\
\hline Harmony & 3.28 & $0.020^{*}$ & Perspective drawing/model & $0.036^{*}$ \\
\hline Pleasantness & 3.81 & $0.010^{\star}$ & Chips/perspective drawing & $0.013^{*}$ \\
\hline \multirow[t]{3}{*}{ Comfort } & 15.56 & $0.000^{\star *}$ & Chips/perspective drawing & $0.000^{\star *}$ \\
\hline & & & Chips/model & $0.002^{*}$ \\
\hline & & & Perspective drawing/model & $0.000^{\star *}$ \\
\hline \multirow[t]{3}{*}{ Spaciousness } & 10.80 & $0.000^{\star *}$ & Chips/perspective drawing & $0.000^{\star *}$ \\
\hline & & & Abstract graphic/perspective drawing & $0.000^{* *}$ \\
\hline & & & Perspective drawing/model & $0.028^{*}$ \\
\hline \multirow[t]{2}{*}{ Dynamism } & 4.78 & $0.003^{*}$ & Chips/model & $0.008^{\star}$ \\
\hline & & & Perspective drawing/model & $0.042^{*}$ \\
\hline Excitement & 0.70 & 0.550 & & \\
\hline \multirow{3}{*}{ Introversion } & 10.79 & $0.000^{\star \star}$ & Chips/abstract graphic & $0.045^{\star}$ \\
\hline & & & Abstract graphic/perspective drawing & $0.000^{\star *}$ \\
\hline & & & Abstract graphic/model & $0.002^{*}$ \\
\hline
\end{tabular}

pleasing/unpleasing and harmonious/discord, and seems to be "evaluation." The second factor gathers the adjective pairs static/dynamic, calming/exciting and introverted/ extroverted, and seems to be "activity." The third factor gathers the adjective pairs spacious/confined and comfortable/uncomfortable. For the abstract compositions, the first factor accounted for $28 \%$ of the variance, the second factor $26 \%$ and the third factor $25 \%$. The first factor gathers the adjective pairs static/dynamic, calming/exciting and introverted/extroverted. The second factor gathers the adjective pairs pleasing/unpleasing and harmonious/discord, and the third factor gathers the adjective pairs spacious/confined and comfortable/uncomfortable.

The perspective drawings and the models showed nearly the same order of factors and patterns of gathering adjectives. Differences are observed only in the percentages of the variances and in the order of two adjectives in the second factor. For the perspective drawings, the first factor accounted for $38 \%$ of the variance and the second factor $30 \%$. For the models, the first factor accounted for $39 \%$ of the variance and the second factor $29 \%$. The first factor gathers the adjective pairs comfortable/uncomfortable, pleasing/unpleasing, harmonious/discord and spacious/confined, and the second factor gathers the adjectives static/dynamic, calming/exciting and introverted/ extroverted.

iii. Stepwise multiple regression was performed in order to see whether the same colour and scheme characteristics predict the semantic ratings of colour schemes presented in different media. Table VI shows the predictors of adjectives for each medium.

Results showed that different colour and scheme characteristics are determinative over the ratings of adjectives when the medium changes. In only one case (pleasing/ unpleasing presented in perspective drawings) are no significant predictors observed. The only consistency is between the predictors of ratings of the adjective pair observed for introverted/extroverted. For this adjective pair, Value was entered first and explained 6\%,8\%, 15\%, and $7 \%$ of the variance in introversion for colour chips, abstract graphics, perspective drawings and models, respectively. Other characteristics explained a further percentage not greater than $2 \%$. In 17 of the total 28 cases, the explained percentage of variance ( $R$ square change) appeared at under $10 \%$.

\section{DISCUSSION}

The present research was conducted to explore the architectural/spatial colouring process by investigating the consistency of semantic ratings of four sequential stages of the architectural colour design process, namely, colour chips/samples, abstract compositions, perspective drawings and 3D models. The results of the study have contributions not only for previous research comparing colour samples and applied colours in different fields of design, but also for propounding a methodology for the architectural colour design process.

From the findings it can be concluded that abstract media can be used as a tool for initial colour-design decisions in architecture, however, scrutinizing colour design through a media simulating contextual characteristics and threedimensional relations is inevitable during the progression of the architectural colour-design process. This work contradicts the research indicating a strong correspondence between colour chips and contextualized colours (e.g., Taft, $^{21}$ Sivik, ${ }^{22}$ Hogg et al. ${ }^{25}$ ), the results showed that the colour chips cannot be considered as efficient representatives of architectural colouring. Significant associations observed between the semantic ratings of abstract graphics, and perspective drawings and models means that the abstract graphics were evaluated more similarly with the contextual media. Therefore, it can be said that abstract compositions presenting both proportional relations and adjacency of colours seem to be more appropriate tools than colour chips.

There is an overall significant medium effect for six of the seven adjectives: only for "excitement" is no medium effect observed (see the results of multiple comparisons in ANOVA-Table IV). This observation may indicate that this construct is evaluated independently from the 


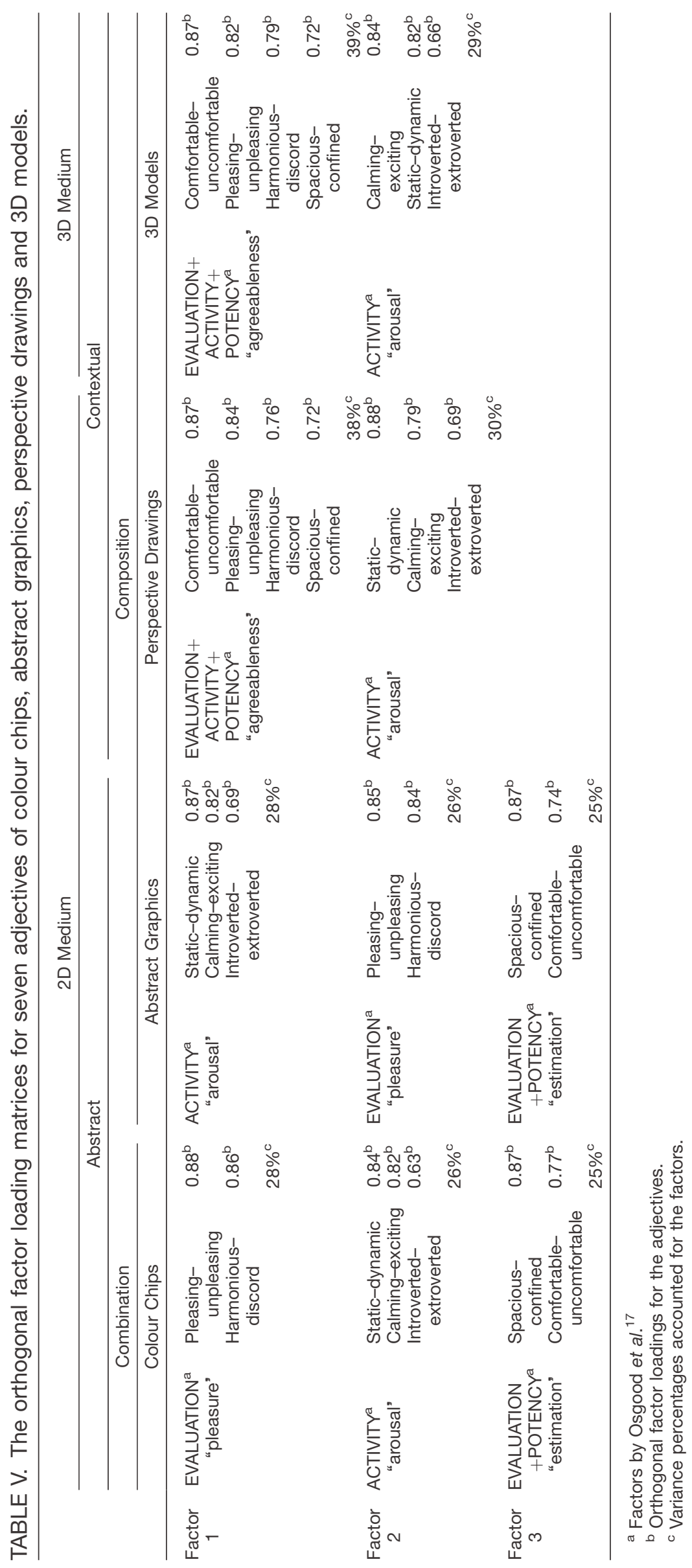


TABLE VI. Stepwise multiple regression of predictors of adjectives (only significant predictors are included).

\begin{tabular}{|c|c|c|c|c|c|c|c|c|c|c|c|c|}
\hline & \multicolumn{9}{|c|}{ 2D Medium } & \multicolumn{3}{|c|}{ 3D Medium } \\
\hline & \multicolumn{6}{|c|}{ Abstract } & \multicolumn{6}{|c|}{ Contextual } \\
\hline & \multicolumn{3}{|c|}{ Combination } & \multicolumn{9}{|c|}{ Composition } \\
\hline & \multicolumn{3}{|c|}{ Colour chips } & \multicolumn{3}{|c|}{ Abstract graphics } & \multicolumn{3}{|c|}{ Perspective drawings } & \multicolumn{3}{|c|}{ Model } \\
\hline & variable & $\begin{array}{c}R \text { sq } \\
\text { change }\end{array}$ & $\begin{array}{l}\text { Sig. } \\
\text { of } t\end{array}$ & variable & $\begin{array}{c}R \text { sq } \\
\text { change }\end{array}$ & Sig. of $t$ & variable & $\begin{array}{c}R \text { sq } \\
\text { change }\end{array}$ & Sig. of $t$ & variable & $\begin{array}{c}R \text { sq } \\
\text { change }\end{array}$ & Sig. of $t$ \\
\hline \multirow[t]{3}{*}{ Harmony } & V & 0.068 & 0.000 & V & 0.062 & 0.000 & C & 0.038 & 0.000 & $\mathrm{schH}$ & 0.060 & 0.000 \\
\hline & C & 0.058 & 0.000 & $\mathrm{H}$ & 0.048 & 0.001 & & & & C & 0.051 & 0.000 \\
\hline & schC & 0.012 & 0.020 & C & 0.017 & 0.016 & & & & $\operatorname{sch} C$ & 0.015 & 0.012 \\
\hline \multirow[t]{2}{*}{ Pleasantness } & V & 0.075 & 0.000 & V & 0.076 & 0.000 & & & & schH & 0.027 & 0.003 \\
\hline & C & 0.026 & 0.001 & $\mathrm{H}$ & 0.016 & 0.008 & & & & $\mathrm{H}$ & 0.015 & 0.014 \\
\hline \multirow[t]{4}{*}{ Comfort } & C & 0.123 & 0.000 & $\mathrm{H}$ & 0.133 & 0.000 & V & 0.062 & 0.000 & C & 0.108 & 0.000 \\
\hline & $\operatorname{schC}$ & 0.024 & 0.001 & $\mathrm{C}$ & 0.012 & 0.020 & $\operatorname{sch} C$ & 0.058 & 0.000 & $\mathrm{H}$ & 0.034 & 0.000 \\
\hline & & & & & & & $\mathrm{schH}$ & 0.042 & 0.000 & & & \\
\hline & & & & & & & $\mathrm{H}$ & 0.011 & 0.025 & & & \\
\hline \multirow{5}{*}{ Spaciousness } & C & 0.110 & 0.000 & $\mathrm{H}$ & 0.139 & 0.000 & V & 0.298 & 0.000 & V & 0.129 & 0.000 \\
\hline & $\mathrm{H}$ & 0.042 & 0.000 & V & 0.094 & 0.000 & $\operatorname{sch} C$ & 0.015 & 0.003 & C & 0.048 & 0.000 \\
\hline & & & & $\mathrm{C}$ & 0.030 & 0.000 & schH & 0.015 & 0.004 & $\mathrm{H}$ & 0.020 & 0.004 \\
\hline & & & & & & & & & & $\mathrm{schH}$ & 0.016 & 0.004 \\
\hline & & & & & & & & & & $\operatorname{sch} C$ & 0.016 & 0.007 \\
\hline \multirow[t]{4}{*}{ Dynamism } & $\operatorname{sch} C$ & 0.063 & 0.000 & C & 0.051 & 0.000 & V & 0.135 & 0.000 & V & 0.069 & 0.000 \\
\hline & V & 0.029 & 0.003 & schH & 0.036 & 0.000 & C & 0.107 & 0.000 & C & 0.030 & 0.000 \\
\hline & $\mathrm{schH}$ & 0.015 & 0.011 & V & 0.028 & 0.001 & $\mathrm{schH}$ & 0.018 & 0.002 & $\mathrm{schH}$ & 0.024 & 0.002 \\
\hline & & & & & & & & & & $\operatorname{sch} C$ & 0.021 & 0.003 \\
\hline \multirow[t]{5}{*}{ Excitement } & C & 0.044 & 0.000 & schH & 0.084 & 0.000 & C & 0.149 & 0.000 & schH & 0.066 & 0.000 \\
\hline & $\mathrm{schH}$ & 0.032 & 0.000 & C & 0.057 & 0.000 & V & 0.038 & 0.000 & V & 0.047 & 0.000 \\
\hline & schV & 0.011 & 0.035 & & & & schH & 0.015 & 0.007 & schV & 0.044 & 0.000 \\
\hline & & & & & & & & & & C & 0.039 & 0.000 \\
\hline & & & & & & & & & & $\operatorname{sch} C$ & 0.009 & 0.034 \\
\hline \multirow[t]{4}{*}{ Introversion } & V & 0.060 & 0.000 & V & 0.082 & 0.000 & V & 0.154 & 0.000 & V & 0.066 & 0.000 \\
\hline & & & & schH & 0.020 & 0.003 & & & & $\mathrm{H}$ & 0.021 & 0.003 \\
\hline & & & & $\mathrm{H}$ & 0.019 & 0.004 & & & & schH & 0.017 & 0.007 \\
\hline & & & & C & 0.018 & 0.005 & & & & schC & 0.017 & 0.008 \\
\hline
\end{tabular}

Colour characteristics; H: Hue; V: Value; C: Chroma.

Scheme characteristics; schH: similarity/contrast in Hue; schV: similarity/contrast in Value; schC: similarity/contrast in Chroma.

characteristics of the medium. According to Scheffe's range test, the medium effect mostly differentiates abstract (chips and abstract graphics) and contextual (perspective drawings and model) visualizations. It can therefore be said that the subjects' criteria for evaluating abstract media differed from their criteria for contextual situations. Between the perspective drawing and the three-dimensional model, a significant medium effect was observed for "spaciousness," "comfort" and "dynamism." This finding may indicate that evaluations of these constructs are more sensitive to a 3D appearance of the colour scheme and/or architectural/spatial aspects.

Results of the factor analysis support the finding that abstract media is being evaluated differently than contextual situations. For the colour chips and the abstract compositions, "harmony" and "pleasure" extracted a factor different than "spaciousness" and "comfort" did; however these four constructs extracted a single factor for the perspective drawings and the models. The constructs "harmony," "pleasantness," "spaciousness" and "comfort" are accounted for as the first factor for contextualized media (perspective drawing and model) and this factor seems to be related to the "agreeableness/appropriateness" of the colour schemes for the defined context. This indicates that in an architectural/spatial context, constructs related to architectural/spatial quality cannot be separated from ones related to the sense of taste or the factor of evaluation ${ }^{20}$ $\left(\right.$ pleasure $^{27}$ ) (see the results of factor analysis-Table V). This finding also supports previous research ${ }^{23,28}$ by revealing the distinctive nature of spatial colour. For the abstract media, "spaciousness" and "comfort" are accounted for as the last factor. Different from "harmony" and "pleasure," these two constructs are seemingly the determinants of architectural/spatial quality and relevant to an "estimation" for architectural/spatial use of colours.

The extraction of "harmony" and "pleasure" for always the same factor recalls the hypothesis by $\mathrm{Ou}$ and Lou': "there is a strong link between colour harmony and the emotion pleasantness" and "a harmonious combination can always give viewers a pleasing impression." However in consideration of the subject group (interior architecture students), this finding cannot be generalized.

In all the four media, the constructs "dynamism," "excitement," and "extroversion" are always accounted for together. This result indicates that the evaluations of these constructs are related regardless of the media in which the colour scheme is presented. They also seem to be related to the factor of activity ${ }^{20}$ (arousal ${ }^{27}$ ) or stimulation (see the results of factor analysis-Table V). This implies that activity ratings are largely independent from the media and/or the spatial context. 
Results of the multiple regression analysis showed that different colour and scheme characteristics are determinative over the ratings of adjectives, however, the percentages explain a maximum total of $30 \%$ in the variance of evaluation of constructs. These results (given in Table VI) indicate that colour-combination emotions, and media effects on their evaluation have complex interrelations and inner dynamics, which are not studied within the scope of this research. Nevertheless, some rough interpretations can be made about the general pattern. According to the results, colour characteristics (dominant Hue, dominant Value and dominant Chroma) seem to be determinant for the evaluation of abstract media while scheme characteristics (similarity or contrast in Hue, similarity or contrast in Value and similarity or contrast in Chroma) appear more effective for the evaluations of contextual media (especially for 3D models). These observations strengthen the difference between abstract and contextualized media used as different visualization techniques in the architectural colour design process.

The overall findings of this research have implications for colour designers who work on architectural/spatial colouring. In architecture, colour chips can be used for initial colour design decisions, however during the colour design process the initial decisions have to be reconsidered by using other media simulating complex colour and colourspace relations. Abstract compositions presenting both proportional relations and adjacency of colours seem to be more appropriate representatives of colour-design compared to colour chips. Perspective drawings may serve to visualize architectural/spatial configuration of colourdesign, but three dimensional appearance of colour combination can only be observed on 3D models. Thus it can be concluded that colour chips, abstract compositions, perspective drawings and 3D models may be used as sequential media during the progression of the process.

Light is a very important integral of colour perception and spatial evaluation. Lighting types (natural and artificial light) and illumination levels do also affect evaluations of colour schemes presented in 3D media. ${ }^{29}$ Examining the effects of different lighting conditions (e.g., different lighting qualities, quantities and patterns), working with other simulation techniques (e.g., computerized media, 3D models at larger or full-scale, and real-life environment) and different subject groups (e.g., designers/architects and nondesigners/architects) may offer different results that could be compared with the ones of this study.

1. Green-Armytage P. The value of knowledge for colour design. Color Res Appl 2006;31:253-269.

2. Lenclos JP. Living in colour. In: Porter T, Mikellides B, editors. Colour for Architecture. New York: Van Nostrand Reinhold; 1976. p 73-77.

3. Pile JF. Color in Interior Design. New York: McGraw Hill; 1997.
4. Ünver R, Öztürk LD. An example of facade colour design of mass housing. Color Res Appl 2002;27:291-299.

5. Smith D. Environmental colouration and/or the design process. Color Res Appl 2003;28:360-365.

6. Mikellides B. Conflicting experience of colour space. In: Simon JG, editor. In: Prooceedings of the Forth IAPC Congress. Belgium: Louvain La Neuve; 1979. Vol. 2.

7. Janssens J, Mikellides B. Color research in architectural educationA cross-cultural explorative study, Color Res Appl 1998;23:328334.

8. Akbay S. An analysis of basic design students' intuitive and analytic attitudes in colour decisions (MFA dissertation). Bilkent University, Ankara, Turkey, 2003.

9. Ou L-C, Lou MR. A colour harmony model for two-colour combinations. Color Res Appl 2006;31:191-204.

10. Arnheim R. Art and Visual Perception. Berkeley: University of California Press; 1974.

11. Birren F. Principles of Color. Pennsilvania: Schiffer Publ. Ltd; 1987.

12. Itten J. The Elements of Color. New York: Van Nostrand Reinhold; 1970.

13. Doyle ME. Color Drawing. New York: Van Nostrand Reinhold; 1981.

14. De Grandis L. Theory and Use of Color. New York: Prentice HallAbrams; 1986.

15. Burchett KE. Color harmony. Color Res Appl 2002;27:38-31.

16. Hård A, Sivik L. A theory of colours in combination-A descriptive model related to the NCS color-order system. Color Res Appl 2001;26:4-28.

17. Nemcsics A. Experimental determination of laws of color harmony. I. Harmony content of different scales with similar hue. Color Res Appl 2007;32:477-488.

18. Ou L-C, Lou MR, Woodcock A, Wright A. A study of colour emotion and colour preference. II. Colour emotions for two colour combinations. Color Res Appl 2004;29:292-298.

19. Ural SE. Color in Architecture: Effects of Color Dynamics on Coloring Artificial Environments ( $\mathrm{PhD}$ dissertation). Karadeniz Technical University, Trabzon, Turkey, 1995.

20. Osgood CE, Suci GJ, Tannenbaum PH. The Measurement of Meaning. Urbana: University of Illinois Press; 1957.

21. Taft C. Color meaning and context: Comparison of semantic ratings of colors on samples and objects. Color Res Appl 1997;22:40-50.

22. Sivik L. Color meaning and perceptual color dimensions: A study of exterior colors. Göteborg Psychol Rep 1974;4:1-24.

23. Billger M. Colour combination. Effects in experimental rooms. Color Res Appl 1999;22:230-242.

24. Destefani LRG, Whitfield TWA. Esthetic decision-making: How do people select colours for real settings? Color Res Appl 2008;33:55-60.

25. Hogg J, Goodman S, Porter T, Mikellides B, Preddy DE. Dimensions and determinants of judgements of colour samples and a simulated interior space by architects and non-architects. Br J Psychology 1979;70:231-242.

26. Stahre B, Hårleman M, Billger M. Colour Emotions in Larger and Small Scale. In: Prooceedings of the AIC Interim Meeting: Colour and Paints, Brazil, 2004.

27. Mehrabian A. Pleasure-arousal-dominance: A general framework for describing and measuring individual differences in temperament. Curr Psychol 1996;14:261-292.

28. Hårleman M, Werner I-B, Billger M. Significance of colour on room character: Study on dominantly reddish and greenish colours in north- and south-facing rooms. Colour Des Creativity 2007;1:115.

29. Ural S, Olguntürk N, Yilmazer S. Qualitative and quantitative evaluations of colour schemes on 2D graphics, 3D models and artificial lighting alternatives (in Turkish), 6. In: Ulusal Aydnlatma Kongresi (6th National Lighting Congress), Istanbul, Türkiye, 2006. 